\title{
Estimação da taxa de cruzamento da aceroleira com base em dados isoenzimáticos ${ }^{(1)}$
}

\author{
Ricardo Lopes ${ }^{(2)}$, Cláudio Horst Bruckner ${ }^{(3)}$ e Maria Teresa Gomes Lopes ${ }^{(2)}$
}

\begin{abstract}
Resumo - A aceroleira (Malpighia emarginata) é uma espécie da família Malpighiaceae que produz frutos com alto conteúdo de vitamina $\mathrm{C}$. O crescimento da demanda da acerola e de seus derivados causou a procura de variedades melhoradas, para expansão dos plantios comerciais. O objetivo deste trabalho foi obter informações sobre o sistema de cruzamento de um pomar de aceroleiras localizado em Visconde do Rio Branco, MG. Dez famílias oriundas de sementes de polinização natural foram genotipadas usando-se sete locos izoenzimáticos (Idh, Mdh-1, Mdh-2, Pgm-1, Pgm-2, Est-1 e Pod), e os dados foram submetidos a análise pelo modelo misto de cruzamento. A estimativa da taxa de cruzamento da população utilizando todos os locos simultaneamente não diferiu estatisticamente de 1,00. Nas famílias, as estimativas variaram de 0,68 a 1,00, e duas diferiram significativamente de 1,00. Conclui-se que a aceroleira é predominantemente alógama e que a taxa de cruzamento varia entre famílias, fato que deve ser levado em consideração em programas de melhoramento e conservação de germoplasma.
\end{abstract}

Termos para indexação: marcadores genéticos, genótipos, identificação, reprodutividade.

\section{Estimation of outcrossing rates in acerola (Malpighia emarginata) based on isoenzymic data}

\begin{abstract}
Acerola (Malpighia emarginata) is a species of Malpighiaceae family that produces fruits with high vitamin $\mathrm{C}$ content. The increasing demand for acerola and its derived products caused the search for improved varieties to expansion of commercial plantation. The objective of this work was to gain information about a breeding system from an acerola population located in Visconde do Rio Branco, MG, Brazil. Ten families originating from seeds of a natural pollination were used. Plants were assayed for seven isoenzymatic loci (Idh, Mdh-1, Mdh-2, Pgm-1, Pgm-2, Est-1 and Pod) and the data were analyzed using a mixed mating model. Multilocos outcrossing rate estimate for population did not differ statistically from 1.00, whereas estimates ranged from 0.68 to 1.00 between families, and two estimates were significantly different from 1.00 . Acerola is predominately allogamous and outcrossing rate varied between families; this fact should be taken into consideration in further breeding programs and germoplasm conservation.
\end{abstract}

Index terms: genetic markers, genotypes, identification, reproductive performance.

\section{Introdução}

A propagação natural da aceroleira (Malpighia emarginata) ocorre por via sexuada; porém, segundo Bosco et al. (1995), ainda não se definiu se a

(1) Aceito para publicação em 24 de abril de 2001.

Extraído da Dissertação de Mestrado apresentada pelo primeiro autor à Universidade Federal de Viçosa (UFV), Viçosa, MG.

(2) Escola Superior de Agricultura Luiz de Queiroz, Dep. de Genética, Av. Pádua Dias, 11, CEP 13418-900 Piracicaba, SP. E-mail: rlopes@carpa.ciagri.usp.br, mtglopes@carpa.ciagri.usp.br

(3) UFV, Dep. de Fitotecnia, CEP 36571-000 Viçosa, MG. E-mail: bruckner@mail.ufv.br aceroleira é uma planta autógama, alógama, ou autógama com alta taxa de alogamia. A flor da aceroleira apresenta algumas características de espécie autógama - flor completa, amadurecimento simultâneo do androceu e gineceu, ausência de barreiras físicas que impeçam ou dificultem o recebimento do pólen no estigma da mesma flor - e é comum a produção de frutos em plantas isoladas em quintais. Alguns fatores sugerem que a espécie não seja predominantemente autógama. Nos plantios comerciais originados de mudas obtidas por via sexuada, é observada grande variabilidade fenotípica, sugerindo a ocorrência de segregação e recombinação gênica (Paiva et al., 1999). A partir da autopolinização ma- 
nual e de botões florais protegidos foi obtida baixa ou nula fixação de frutos sendo constatada maior fixação de frutos quando ocorreu polinização natural e polinização cruzada manual (Lopes et al., 2000).

O sistema de cruzamento é o ponto principal na análise da organização genética das populações, pois ao determinar os padrões pelos quais os gametas são transportados de geração para geração exerce um controle primário sobre as distribuições das freqüências genotípicas e afeta o potencial de recombinação das populações, moderando ou acelerando a taxa de produção de novas combinações gênicas e a perda de algumas já existentes (Allard, 1971).

Segundo Shaw \& Allard (1982), os sistemas de cruzamento em plantas geralmente são descritos pelo modelo misto de cruzamento, no qual se determina a proporção de progênies provenientes de autofecundação (s) e de fecundação cruzada $(\mathrm{t}=1-\mathrm{s})$. As estimativas dos parâmetros do sistema misto de cruzamento, taxa de cruzamento $(\mathrm{t})$ e taxa de autofecundação (s) são necessárias para que sejam utilizadas estratégias adequadas na obtenção de variedades melhoradas e se avalie a viabilidade de tais estratégias, definindo o tipo de variedade a ser recomendada para cultivo comercial (linhagens, híbridos, clones).

As inferências sobre a maneira como ocorrem os cruzamentos podem ser feitas a partir da distribuição de marcadores genéticos nas famílias, de maneira especial os co-dominantes, classe em que se enquadram as isoenzimas. Estudos do sistema de cruzamento baseados em dados isoenzimáticos foram realizados em diversas espécies perenes, como Eucalyptus urophylla (House \& Bell, 1994), Acacia nilotica (Mandal \& Ennos, 1995), Fagus sylvatica (Rossi et al., 1996), Pinus sylvestris (Burczyk, 1998), Pterocarpus macrocarpus (Liengsiri et al., 1998), Dryobalanops aromatica (Lee, 2000) e Eucalyptus marginata (Millar et al., 2000), e também em inúmeras espécies anuais.

As principais vantagens do uso de marcadores isoenzimáticos na estimação dos parâmetros do sistema de cruzamento estão relacionadas ao fato de serem geralmente marcadores co-dominantes, permitirem a análise ainda no estádio de plântula e por serem as populações de plantas, em geral, polimórficas para vários locos isoenzimáticos (Brown \& Allard, 1970). Marcadores co-dominantes permitem a identificação do genótipo (homozigoto ou heterozigoto) sem a necessidade de testes de progênies, e além disso, os processos de estimação baseados nesse tipo de marcadores são mais eficientes. A possibilidade de caracterização das plântulas logo após a germinação diminui o tempo necessário para se obter informações a respeito do sistema de cruzamento. O número de locos analisados está relacionado à precisão das estimativas. Em geral, analisando-se um maior número de locos pode-se atingir estimativas mais precisas. Além disso, é possível obter e comparar taxas de cruzamento verificadas a partir de locos individuais e de locos múltiplos para mais informações sobre a endogamia.

O objetivo deste trabalho foi estimar os parâmetros do sistema de cruzamento da aceroleira.

\section{Material e Métodos}

As análises foram feitas utilizando-se 10 famílias, oito compostas de 16 plantas, uma de 18 plantas e uma de 12 plantas. As matrizes (genitores maternos) foram tomadas aleatoriamente no pomar da Universidade Federal de Viçosa, que possui aproximadamente 130 plantas, localizado no Município de Visconde do Rio Branco, MG. Em março de 1998, frutos maduros foram coletados e despolpados em água corrente, e as sementes secadas à sombra. Após a secagem, as sementes foram semeadas em caixas com vermiculita, onde permaneceram até a germinação; quando apareceu o primeiro par de folhas definitivas, as plântulas foram transplantadas para copos de plástico de $300 \mathrm{~mL}$, preenchidos com substrato composto de $50 \%$ de terra, $25 \%$ de areia grossa e $25 \%$ de esterco e mantidos em casa de vegetação.

Na genotipagem multilocos das progênies, a amostragem das folhas para obtenção do extrato bruto, utilizado nas corridas eletroforéticas, foi feita quando as plantas apresentavam aproximadamente cinco meses de idade. Foram utilizados sete locos isoenzimáticos: isocitrato desidrogenase (Idh), malato desidrogenase 1 e 2 (Mdh-1 e Mdh-2), fosfoglucomutase 1 e 2 (Pgm-1 e Pgm-2), esterase (Est-1) e peroxidase (Pod). Os locos foram escolhidos a partir do estudo realizado por Lopes (1999), por terem 
apresentado boa definição, serem polimórficos, não apresentarem sobreposição de bandas, alelos nulos ou outros artefatos e permitirem a interpretação segura dos padrões de segregação necessária para a genotipagem das progênies.

Os genótipos foram representados por números que correspondem aos alelos apresentados pelo indivíduo. $\mathrm{O}$ alelo número 1 corresponde à banda de maior migração na região do gel referente ao loco; o alelo número 2 corresponde à segunda banda de maior migração, e assim sucessivamente. Nos casos em que não foi possível determinar o genótipo do indivíduo, utilizou-se o número 0 .

Após a genotipagem multilocos das famílias, foi verificada a proporção de indivíduos provenientes de cruzamento não ambíguo de cada uma das famílias, ou seja, indivíduos portadores de um ou mais alelos não apresentados pelo genitor feminino.

No cálculo das estimativas dos parâmetros do sistema de cruzamento, utilizou-se o programa MLTR (Ritland, 1990). O método de estimação utilizado pelo programa é baseado em equações de máxima verossimilhança (Ritland, 1983). Esse processo de estimação é empregado quando os genótipos dos genitores femininos são conhecidos e as freqüências gênicas no conjunto de pólen são desconhecidas. Pelo fato de as progênies envolverem duas ou mais classes genotípicas de genitores femininos, foi possível a estimação simultânea da taxa de cruzamento e das freqüências alélicas no conjunto de pólen. As estimativas de máxima verossimilhança foram encontradas por meio da derivação do logaritmo da função de verossimilhança de forma que esta tenda a zero, ou seja, maximizando a função.

Foram obtidas as estimativas dos seguintes parâmetros: a) $T_{m}=$ taxa de cruzamento com base em multilocos, encontradas a partir de genótipos compostos, para a população e para as famílias individualmente; b) $\mathrm{T}_{\text {smédio }}=$ taxa de cruzamento uniloco média, para comparação com a taxa de cruzamento com base em multilocos; c) F = índice de fixação; d) p = freqüência gênica no conjunto de pólen e óvulo; e) variâncias das estimativas.

As variâncias das estimativas foram encontradas pelo método Bootstrap, cujas plantas dentro das famílias foram a unidade de reamostragem, obtendo-se, então, os desvios-padrões a 95\% de intervalo de confiança e, com isso, a significância dos valores das taxas de cruzamento.

\section{Resultados e Discussão}

A proporção de progênies provenientes de cruzamento não ambíguo foi de 37,5\% nas famílias 2, $8 \mathrm{e}$ $9 ; 66,7 \%$ na família $3 ; 87,5 \%$ nas famílias 6 e 10;93,75\% nas famílias 4, 5 e 7; e 94,4\% na família 1. Segundo Shaw et al. (1981), a probabilidade de observação direta de cruzamento, isto é, da ocorrência de cruzamentos não ambíguos, aumenta com o número de locos, o número de alelos por loco e também com as freqüências dos alelos mais próximas. Assim, a variação da proporção de cruzamentos não ambíguos observada entre as famílias é decorrente do número de locos em homozigose apresentado pelo genitor feminino e da freqüência, no conjunto de pólen, dos alelos que este não apresenta.

Os valores das estimativas de máxima verossimilhança dos parâmetros do sistema de cruzamento, obtidos pelo método de maximização da esperança (EM), foram sempre menores em valor absoluto do que os obtidos pelo método de Newton-Ranpson (NR) (Tabela 1). O método EM limita as estimativas da taxa de cruzamento ao intervalo 0 - 1 , enquanto pelo método NR essas estimativas podem variar de 0-2. Ambos os métodos apresentam estimativa da taxa de cruzamento com base em multilocos não diferindo significativamente de 1 , evidenciando que na população as freqüências genotípicas na geração avaliada estão de acordo com o esperado em uma população alógama. Em relação à taxa de cruzamento uniloco média, a estimativa obtida pelo método $\operatorname{EM}(0,945)$ foi inferior à encontrada pelo método NR $(1,004)$. O valor negativo da estimativa do índice de fixação obtido pelo método NR bem como o valor zero pelo método EM indicam a não-ocorrência de endogamia na população.

A comparação dos parâmetros $\mathrm{t}_{\mathrm{m}}$ e $\mathrm{t}_{\text {smédia }}$ evidencia a ausência de cruzamento entre parentes, uma vez que a diferença entre eles é praticamente zero (Tabela 1). De acordo com Shaw \& Allard (1982), a diferença positiva indica a ocorrência de cruzamento correlacionado (endogamia biparental), pois, quando ocorre endogamia decorrente do cruzamento entre parentes em adição à autofecundação, espera-se que as estimativas multilocos de $\mathrm{t}$ sejam maiores do que as estimativas uniloco. Segundo Robinson (1998), a diferença entre $t_{m}$ e $t_{\text {smédia }}$ também pode ser decorrente da heterogeneidade nas freqüências alélicas durante o período de florescimento (resultantes da defasagem no florescimento ou das dife- 
renças em fecundidade entre as plantas). Portanto, a diferença não-significativa encontrada revela que a freqüência alélica no conjunto de pólen que efetivamente contribuiu na formação dos novos zigotos distribuiu-se uniformemente sobre os genitores femininos.

As estimativas de máxima verossimilhança da taxa de cruzamento das famílias, com base em multilocos, obtidas pelo método EM variaram de 0,68 a 1,00, e de 0,68 a 2,00, pelo método NR (Tabela 1). Junghans et al. (1998) afirmaram que as estimativas da taxa de cruzamento maiores do que 1,00 não devem ser consideradas pelo seu valor nominal nem descartadas, mas sim interpretadas como $100 \%$ de cruzamento. As taxas de cruzamento das famílias 3 e 9 obtidas pelo método EM diferiram significativamente de 1,00, indicando a ocorrência de autofecundação nessas famílias. Na família 2, verificou-se a não-convergência da estimativa de máxima verossimilhança pelo método NR $\left(\mathrm{t}_{\mathrm{m}}=2,00\right)$. Segundo Ritland \& Jain (1981), valores da taxa de cruzamento iguais a 2,00 ocorrem porque a distribuição dos genótipos nas progênies não permite a convergência das estimativas a um valor único, e isto indica que está ocorrendo uma ou mais violações das pressuposições do modelo.

Tabela 1. Estimativas de máxima verossimilhança da taxa de cruzamento da aceroleira com base em multilocos $\left(t_{m}\right)$ em nível de população e de dez famílias e uniloco média $\left(t_{\text {smédia }}\right)$, índice de fixação $(F)$ e diferença das estimativas de $t_{m}$ e $t_{\text {smédio }}$ da população obtidas pelos métodos auxiliares Newton-Ranpson e maximização da esperança ${ }^{(1)}$.

\begin{tabular}{lcr}
\hline Estimativa & $\begin{array}{c}\text { Método da } \\
\text { maximização da } \\
\text { esperança }\end{array}$ & $\begin{array}{c}\text { Método de } \\
\text { Newton-Ranpson }\end{array}$ \\
\hline $\mathrm{t}_{\mathrm{m}}$ em nível de população & $0,999(0,009)$ & $1,013(0,045)$ \\
$\mathrm{t}_{\text {smédia }}$ em nível de população & $0,945(0,010)$ & $1,004(0,049)$ \\
$\mathrm{F}$ & $0,000(0,000)$ & $-0,421(0,241)$ \\
$\mathrm{t}_{\mathrm{m}} \mathrm{t}_{\text {smédio }}$ & $0,054(0,010)$ & $0,008(0,033)$ \\
$\mathrm{t}_{\mathrm{m}}$ em nível de família & $1,00(0,05)$ & $1,02(0,52)$ \\
1 & $1,00(0,00)$ & $2,00(0,45)$ \\
2 & $0,74(0,18)$ & $0,74(0,50)$ \\
3 & $1,00(0,00)$ & $1,13(0,41)$ \\
4 & $1,00(0,03)$ & $1,14(0,44)$ \\
5 & $1,00(0,05)$ & $1,08(0,47)$ \\
6 & $1,00(0,00)$ & $1,24(0,36)$ \\
7 & $0,99(0,01)$ & $1,71(0,30)$ \\
8 & $0,68(0,18)$ & $0,68(0,44)$ \\
9 & $1,00(0,00)$ & $1,08(0,46)$ \\
10 & &
\end{tabular}

Pesq. agropec. bras., Brasília, v. 37, n. 3, p. 321-327, mar. 2002
De acordo com esses autores, as principais causas da não-convergência das estimativas de máxima verossimilhança são a interpretação incorreta dos genótipos dos genitores femininos, número reduzido de plantas por família, pequeno número de famílias e genótipos maternos apresentando a maioria dos locos em estado de heterozigose.

Para o ajuste dos dados ao modelo, este pressupõe que todos os genótipos maternos cruzam a uma mesma taxa a partir de um conjunto de pólen que se distribui uniformemente sobre a população, ausência de seleção, mutação ou deriva genética, afetando os locos marcadores e segregação mendeliana simples dos alelos em plantas maternais heterozigotas (1:1), tanto para produção de pólen como de óvulo.

Utilizando-se a estatística qui-quadrado para a comparação das proporções esperadas e observadas das classes genotípicas das progênies, é possível detectar algumas violações do modelo misto de cruzamento, caso estas sejam suficientemente expressivas. Tais violações incluem o favorecimento de heterozigotos após o evento de cruzamento, correlações de freqüências gênicas no conjunto de pólen com genótipos maternos durante o cruzamento e taxa de cruzamento dependente do genótipo (Ritland, 1983).

A comparação das freqüências esperadas e observadas das classes genotípicas das progênies, para cada loco individualmente, indica que violações do modelo estão ocorrendo nos locos Idh e Est-1, o que pode ser verificado pela baixa probabilidade da estatística qui-quadrado (Tabela 2). No loco Idh, verifica-se que a freqüência de indivíduos heterozigotos observada apresentou-se superior à esperada nas progênies provenientes das três classes de genótipos dos genitores femininos. Essa observação sugere que a seleção favorecendo o heterozigoto pode estar ocorrendo nesse loco. Oliveira (1997) apresentou relações entre genótipos heterozigotos da enzima IDH e a alta taxa de germinação em acessos de Bidens pilosa.

De acordo com Ritland (1983), freqüentemente são encontrados desvios significativos das freqüências 
esperadas. $\mathrm{O}$ autor ressaltou que quando vários locos são usados e, entre esses, poucos mostram desvios significativos, não há necessidade de descarte de locos e que as estimativas multilocos tendem a ser robustas diante de violações das pressuposições do modelo. O loco Pgm-2 não foi incluído no teste por apresentar grande número de classes genotípicas com valores esperados abaixo de cinco, decorrente do fato de o sistema apresentar quatro alelos, dois com baixa freqüência associada ao número de progênies analisadas.

Diferenças significativas entre as freqüências alélicas no conjunto de pólen que contribuem efetivamente para o cruzamento e no conjunto de óvulo foram encontradas nos locos Mdh-1 $(\mathrm{P}=1,275) \mathrm{e}$ Pgm-2 ( $\mathrm{P}=3,235)$ (Tabela 3). Nos cinco demais locos, as freqüências alélicas podem ser consideradas homogêneas, indicando que o conjunto de pólen foi uma amostra aleatória das árvores adultas, as quais foram satisfatoriamente representadas pelas árvores maternas.
O teste qui-quadrado também foi aplicado para verificar a homogeneidade do número de plantas heterozigóticas provenientes de genitores femininos homozigóticos de mesmo genótipo (Tabela 4). Quando o cruzamento é aleatório e o conjunto de pólen é distribuído de forma homogênea sobre a população, espera-se que o número de plântulas heterozigotas seja o mesmo em famílias de genitores femininos com mesmo genótipo homozigoto. Não foram encontradas diferenças estatísticas entre o número de plantas heterozigóticas provenientes de genitores femininos de mesmo genótipo homozigoto, o que sugere que as plantas cruzaram a uma mesma taxa (Tabela 4).

Fu et al. (1992) comentaram que violações das pressuposições do modelo considerado resultam em inacurácia das estimativas de $t$ e que pouco se sabe sobre o viés de $t$ resultante das violações das pressuposições. Considerando os resultados obtidos mediante os testes aplicados, pode-se considerar que os dados, embora com alguns indícios de violações, ajustam-se ao modelo aleatório de cruzamento e proporcionaram estimativas robustas dos parâmetros do sistema de cruzamento.

Tabela 2. Proporções genotípicas observadas e esperadas nas progênies de aceroleira e teste $\chi^{2}$ para verificação da hipótese de cruzamento aleatório populacional.

\begin{tabular}{|c|c|c|c|c|c|c|c|c|c|c|}
\hline \multirow[t]{3}{*}{$\operatorname{Loco}^{(1)}$} & \multirow[t]{3}{*}{ Progênie } & \multicolumn{6}{|c|}{ Genitor feminino } & \multirow[t]{3}{*}{$\chi^{2}$} & \multirow[t]{3}{*}{ GL } & \multirow[t]{3}{*}{ Probabilidade $(\%)$} \\
\hline & & \multicolumn{2}{|c|}{11} & \multicolumn{2}{|c|}{12} & \multicolumn{2}{|c|}{22} & & & \\
\hline & & Obs. & Esp & Obs. & Esp & Obs. & Esp & & & \\
\hline \multirow[t]{3}{*}{ Idh } & 11 & 19,00 & 26,50 & 23,00 & 20,14 & - & - & & & \\
\hline & 12 & 31,00 & 23,50 & 45,00 & 38,00 & 18,00 & 16,96 & & & \\
\hline & 22 & - & - & 8,00 & 17,86 & 15,00 & 15,04 & 11,790 & 6 & 6,68 \\
\hline \multirow[t]{2}{*}{ Mdh-1 } & 12 & - & - & - & - & 34,00 & 33,54 & & & \\
\hline & 22 & - & - & - & - & 122,00 & 122,46 & 0,008 & 1 & 92,87 \\
\hline \multirow[t]{3}{*}{ Mdh-2 } & 11 & 89,00 & 89,83 & 20,00 & 17,34 & - & - & & & \\
\hline & 12 & 25,00 & 24,17 & 19,00 & 22,00 & - & - & & & \\
\hline & 22 & - & - & 5,00 & 4,66 & - & - & 0,840 & 4 & 93,30 \\
\hline \multirow[t]{3}{*}{ Est-1 } & 11 & - & - & 40,00 & 24,83 & - & - & & & \\
\hline & 12 & - & - & 39,00 & 61,00 & 3,00 & 9,36 & & & \\
\hline & 22 & - & - & 43,00 & 36,17 & 20,00 & 13,63 & 25,790 & 4 & 3,49 \\
\hline \multirow[t]{3}{*}{ Pgm-1 } & 11 & 85,00 & 87,05 & 22,00 & 19,34 & - & - & & & \\
\hline & 12 & 23,00 & 20,95 & 23,00 & 24,00 & - & - & & & \\
\hline & 22 & - & - & 3,00 & 4,66 & - & - & 1,244 & 4 & 87,08 \\
\hline \multirow[t]{3}{*}{ Pod } & 11 & - & - & 30,00 & 29,75 & - & - & & & \\
\hline & 12 & - & - & 68,00 & 70,00 & 7,00 & 6,80 & & & \\
\hline & 22 & - & - & 42,00 & 40,25 & 9,00 & 9,20 & 0,145 & 4 & 99,75 \\
\hline
\end{tabular}

${ }^{(1)} \mathrm{Idh}$ : Isocitrato desidrogenase; Mdh-1: Malato desidrogenase 1; Mdh 2: Malato desidrogenase 2; Est-1: Esterase 1; Pgm-1: Fosfoglucomutase 1; Pod: Peroxidase. 
Tabela 3. Estimativas de máxima verossimilhança das freqüências alélicas atribuídas a pólen e óvulo (desvio-padrão entre parênteses) nas progênies de 10 famílias de aceroleira e teste $\chi^{2}$ de heterogeneidades das duas freqüências contribuindo ao conjunto gênico de sementes para sete locos isoenzimáticos ${ }^{(1)}$.

\begin{tabular}{|c|c|c|c|c|c|}
\hline $\operatorname{Loco}^{(2)}$ & Alelo & Conjunto de pólen & Conjunto de óvulo & $\chi^{2}(\mathrm{GL})$ & Probabilidade (\%) \\
\hline \multirow[t]{2}{*}{ Idh } & 1 & $0,530(0,056)$ & $0,550(0,102)$ & $0,128(1)$ & 72,05 \\
\hline & 2 & $0,470(0,056)$ & $0,450(0,102)$ & & \\
\hline \multirow[t]{2}{*}{ Mdh-1 } & 1 & $0,215(0,025)$ & $0,048(0,002)$ & $19,047(1)$ & 1,28 \\
\hline & 2 & $0,785(0,025)$ & $0,952(0,002)$ & & \\
\hline \multirow[t]{2}{*}{ Mdh-2 } & 1 & $0,788(0,024)$ & $0,850(0,072)$ & $2,040(1)$ & 15,32 \\
\hline & 2 & $0,212(0,024)$ & $0,150(0,072)$ & & \\
\hline \multirow[t]{2}{*}{ Est-1 } & 1 & $0,407(0,066)$ & $0,400(0,060)$ & $0,015(1)$ & 90,35 \\
\hline & 2 & $0,593(0,066)$ & $0,600(0,060)$ & & \\
\hline \multirow{2}{*}{ Pgm-1 } & 1 & $0,806(0,032)$ & $0,850(0,073)$ & $1,060(1)$ & 30,32 \\
\hline & 2 & $0,194(0,032)$ & $0,150(0,073)$ & & \\
\hline \multirow[t]{4}{*}{ Pgm-2 } & 1 & $0,401(0,059)$ & $0,381(0,094)$ & $8,781(3)$ & 3,24 \\
\hline & 2 & $0,311(0,062)$ & $0,381(0,151)$ & & \\
\hline & 3 & $0,136(0,057)$ & $0,190(0,084)$ & & \\
\hline & 4 & $0,153(0,037)$ & $0,048(0,002)$ & & \\
\hline \multirow[t]{2}{*}{ Pod } & 1 & $0,425(0,033)$ & $0,450(0,045)$ & $0,198(1)$ & 65,63 \\
\hline & 2 & $0,575(0,033)$ & $0,550(0,045)$ & & \\
\hline
\end{tabular}

(1)Erro-padrão entre parênteses. ${ }^{(2)}$ Idh: Isocitrato desidrogenase; Mdh-1: Malato desidrogenase 1; Mdh-2: Malato desidrogenase 2; Est-1: Esterase 1; Pgm-1: Fosfoglucomutase 1; Pod: Peroxidase.

Tabela 4. Teste $\chi^{2}$ para verificação da homogeneidade do número de plantas heterozigotas provenientes de genitores femininos de mesmo genótipo homozigoto.

\begin{tabular}{|c|c|c|c|c|}
\hline $\operatorname{Loco}^{(1)}$ & Genótipo materno & $\chi^{2}$ & GL & Probabilidade (\%) \\
\hline \multirow[t]{2}{*}{ Idh } & 11 & 0,393 & 2 & 82,16 \\
\hline & 22 & 0,000 & 1 & 100,00 \\
\hline Mdh-1 & 22 & 8,190 & 9 & 41,99 \\
\hline Mdh-2 & 11 & 1,940 & 5 & 85,74 \\
\hline Est-1 & 22 & 2,150 & 1 & 14,26 \\
\hline $\mathrm{Pgm}-1$ & 11 & 4,830 & 6 & 56,58 \\
\hline
\end{tabular}

(1)Idh: Isocitrato desidrogenase; Mdh-1: Malato desidrogenase 1; Mdh-2: Malato desidrogenase 2; Est-1: Esterase 1; Pgm-1: Fosfoglucomutase 1.

\section{Conclusões}

1. A aceroleira demonstra ser uma espécie predominantemente alógama.

2. A taxa de cruzamento natural da aceroleira varia entre famílias.

\section{Referências}

ALLARD, R. W. Princípios de melhoramento genético de plantas. São Paulo: E. Blücher, 1971. 381 p.

BOSCO, J.; BARRETO NETO, M. B.; AGUIAR FILHO, S. P. de; MELO, A. S. de; BAROS, R. V.; MAIA NETO, J. S.; SILVA, J. E. da; NASCIMENTO, R. G. Pesquisa e extensão com acerola na Paraíba. In: SÃO JOSÉ, A. R.; ALVES, R. E. (Ed.). Acerola no Brasil: produção e mercado. Vitória da Conquista: Uesb, 1995. p. 124-129.

BROWN, A. H. D.; ALLARD, R. W. Estimation of the mating system in open-pollinated maize populations using isozyme polymorphisms. Genetics, Baltimore, v. 66, p. 133-145, 1970.

BURCZYK, J. Mating system variation in a Scots pine clonal seed orchard. Silvae Genetica, Frankfurt, v. 47, p. 155-158, 1998.

FU, Y. B.; KNOWLES, P.; PERRY, D. J. Pollen pool heterogeneity in jack pine (Pinus banksiana Lamb.): a problem for estimating outcrossing rates? Theoretical and Applied Genetics, Berlin, v. 83, p. 500-508, 1992.

HOUSE, A. P. N.; BELL, J. C. Isozyme variation and mating system in Eucalyptus urophyla ST Blake. Silvae Genetica, Frankfurt, v. 43, n. 2/3, p. 167-179, 1994.

JUNGHANS, T. G.; PETERS-ROBINSON, I.; BERTOLUCCI, F. L.; ALFENAS, A. C. The use of selfincompatibility in the production of hybrid eucalyptus seed by "Aracruz Celulose" in Brazil. Genetics and Molecular Biology, Ribeirão Preto, v. 21, n. 3, p. 375379, 1998. 
LEE, S. L. Mating system parameters of Dryobalanops aromatica Gaertn. F. (Dipterocarpaceae). Heredity, Oxford, v. 85, n. 4, p. 338-345, 2000.

LIENGSIRI, C.; BOYLE, T. J. B.; YEH, F. C. Mating system in Pterocarpus macrocarpus Kuzs in Thailand. Journal of Heredity, Cary, v. 89, p. 216-221, 1998.

LOPES, R. Polimorfismo, sistema de acasalamento, polinizações, repetibilidade de características do fruto e avaliação de acessos de aceroleira (Malpighia emarginata DC.). Viçosa, MG: UFV, 1999.160 p. Dissertação de Mestrado.

LOPES, R.; BRUCKNER, C. H.; LOPES, M. T. G. Polinização e vingamento de frutos em aceroleira (Malpighia punicifolia L.). Revista Brasileira de Fruticultura, Jaboticabal, v. 22, n. 3, p. 314-317, 2000.

MANDAL, A. K.; ENNOS, E. A. Mating system analysis in a natural population of Acacia nilotica subspecies kraussiana. Forest Ecology and Management, Amsterdam, v. 79, n. 3, p. 235-240, 1995.

MILlAR, M. A.; BYRNE, M.; COATES, D. J.; STUKELY, M. J. C.; McCOMB, J. A. Mating system studies in jarrah, Eucalyptus marginata (Myrtaceae). Australian Journal of Botany, Collingwood, v. 48, n. 4, p. 475-479, 2000.

OLIVEIRA, J. E. Z. Variabilidade isozimática e do teor de óleo em acessos de Bidens pilosa $\mathrm{L}$. Viçosa, MG: UFV, 1997. 72 p. Dissertação de Mestrado.

PAIVA, J. R. de; ALVES, R. E.; CORREA, M. P. F.; FREIRE, F. das C. O.; BRAGA SOBRINHO, R. Seleção massal de acerola em plantio comercial. Pesquisa
Agropecuária Brasileira, Brasília, v. 34, n. 3, p. 505-511, mar. 1999.

RITLAND, K. Estimation of mating systems. In: TANKSLEY, S. D.; ORTON, T. J. (Ed.). Isozymes in plant genetics and breeding. Amsterdam: Elsevier Science, 1983. Part A, p. 289-302.

RITLAND, K. A series of FORTRAN computer programs for estimating plant mating system. Journal of Heredity, Cary, v. 81, p. 235-237, 1990.

RITLAND, K.; JAIN, S. A model for the estimation of outcrossing rate and gene frequencies using $\mathrm{n}$ independent loci. Heredity, Oxford, v. 47, p. 35-52, 1981.

ROBINSON, I. P. Aloenzimas na genética de populações de plantas. In: ALFENAS, A. C. (Ed.). Eletroforese de isoenzimas e proteínas afins: fundamentos e aplicações em plantas e microorganismos. Viçosa, MG: UFV, 1998. p. $329-368$.

ROSSI, P.; VENDRAMIN, G. G.; GIANNINI, R. Estimation of mating system parameters in two Italian natural populations of Fagus sylvatica. Canadian Journal of Forest Research, Ottawa, v. 26, n. 7, p. 11871192, 1996.

SHAW, D. V.; ALLARD, R. W. Estimation of outcrossing rates in Douglas-fir using isozyme markers. Theoretical and Applied Genetics, Berlin, v. 62, p. 113-120, 1982.

SHAW, D. V.; KAHLER, A. L.; ALLARD, R. W. A multilocos estimator of mating system parameters in plant population. Proceedings of the National Academy of Sciences of the United States of America, Washington, v. 78, n. 2, p. 1298-1302, 1981. 